\title{
Nivel de inteligencia emocional en niños y niñas del II ciclo de instituciones educativas de la zona rural durante la pandemia-Puno 2021
}

Level of emotional intelligence in boys and girls of the second cycle of educational institutions in rural areas during the pandemic - Puno 2021

Nível de inteligência emocional em meninos e meninas de II ciclo de instituições de educação na área rural durante a pandêmica - Puno 2021

\author{
Nelly Olga Zela Payi ${ }^{1}$ \\ nzela@unap.edu.pe \\ https://orcid.org/0000-0001-9119-1102 \\ Nancy Chambi Condori ${ }^{1}$ \\ nchambi@unap.edu.pe \\ https://orcid.org/0000-0002-4520-4707
}

Haydee Clady Ticona Arapa
hcticona@unap.edu.pe
https://orcid.org/0000-0003-3963-9218

Juan Francisco Barrionuevo Valero ${ }^{2}$

jbarrionuevo135@gmail.com

https://orcid.org/0000-0002-4245-0435

${ }^{1}$ Universidad Nacional del Altiplano Puno, Puno-Perú

${ }^{2}$ Instituto de Educación Superior Pedagógico Público Puno-Perú

Artículo recibido 10 de agosto 2021, arbitrado y aceptado 30 de octubre 2021 y publicado 28 de enero 2022

\section{RESUMEN}

Es importante mencionar que durante la pandemia del COVID 19, muchas familias tuvieron problemas emocionales, como tristeza, ira, estado depresivo, preocupación. Ésta investigación determinó los niveles de inteligencia emocional y su importancia, la metodología es de tipo descriptivo, diseño no experimental transversal, se aplicó el test de mesquite a 100 encuestados; los resultados demuestran una valoración de 25 puntos, por lo que indica un nivel de Inteligencia emocional elevada, lo que significa que saben reconocer sus emociones y actúan en base a cada situación, nunca dejan de sentir empatía por los demás, por lo que vienen enfrentando momentos de interacción permanente en el campo familiar rural, es así que son conscientes de sus propias capacidades, pueden afrontar las tensiones normales de la vida, pueden trabajar en forma productiva con sus padres y ser fructíferos con los demás, así como ayudar a su comunidad.

Palabras clave: Interacción; Pandemia; Tensión; Tristeza; Zona rural

ABSTRACT

RESUMO

It is important to mention that during the COVID 19 pandemic, many families had emotional problems, such as sadness, anger, depressed state, worry. This research determined the levels of emotional intelligence and its importance, the methodology is descriptive, non-experimental crosssectional design, the mesquite test was applied to 100 respondents; The results show an assessment of 25 points, which indicates a high level of Emotional Intelligence, which means that they know how to recognize their emotions and act based on each situation, they never stop feeling empathy for others, for what they have been facing moments of permanent interaction in the rural family field, that is how they are aware of their own capacities, can cope with the normal stresses of life, can work productively with their parents and be fruitful with others, as well as help their community .

Key words: Interaction; Pandemic; Tension; Sadness; Rural area
É importante mencionar que durante a pandemia de COVID 19, muitas famílias apresentaram problemas emocionais, como tristeza, raiva, estado de depressão, preocupação. Esta pesquisa determinou os níveis de inteligência emocional e sua importância, a metodologia é descritiva, não experimental de delineamento transversal, o teste de algaroba foi aplicado a 100 respondentes; os resultados mostram uma avaliação de 25 pontos, o que indica um alto nível de Inteligência Emocional, o que significa que sabem reconhecer suas emoções e agir com base em cada situação, nunca deixam de sentir empatia pelos outros, pelos momentos que vêm enfrentando. De convivência permanente no campo da família rural, ou seja, estão conscientes das próprias capacidades, conseguem enfrentar as tensões normais da vida, podem trabalhar produtivamente com os pais e ser frutíferos com os outros, bem como ajudar a sua comunidade.

Palavras-chave: Interação; Pandemia; Tensão; Tristeza; Área rural 


\section{INTRODUCCIÓN}

Durante pandemia que se vive en la actualidad los niños y niñas aprenden y amplían sus conocimientos en el campo familiar referente al manejo emocional frente a situaciones adversas, es así que el término de inteligencia emocional, las familias juegan un rol indispensable en la educación y formación de sus menores hijos y más aún en niños y niñas, la que es considerado como indispensable dentro de la formación así como del aprendizaje en valores desde el punto de vista emocional. Goleman (2007), fue uno de los pioneros en el estudio de la Inteligencia Emocional, donde nos señala que consiste en tener reconocer sus propias emociones, así como el manejo e interacción con los demás, la motivación propia de cada una, así como en el reconocimiento de las emociones de los demás, en el establecimiento de emociones positivas con otras personas. Por tanto, se trata de usar las emociones, aprendiendo a reconocer, a entender su forma de actuar, a fin de que éstas nos ayuden a pensar. Asimismo, es importante a que los niños y niñas tengan la oportunidad de que controlen sus emociones para que las relaciones sociales se establezcan dentro de un marco de armonía y madurez emocional.

Según Goleman (2007), define a la Inteligencia emocional como "la capacidad de reconocer, aceptar y canalizar nuestras emociones para dirigir nuestras conductas a objetivos deseados, lograrlo $y$ compartirlos con los demás".

Ello significa que, si uno es capaz de identificar, controlar, gestionar de manera correctiva las emociones de uno mismo, no dejando que te dominen ni te sorprendan, éstas también pueden ser detectadas y actuar de manera oportuna; en consecuencia, se tiene que cuando se están produciendo la relación con los demás se identifican las emociones y te resultarán mucho más gratificantes para canalizar las emociones.

La Inteligencia Emocional puede dividirse en cuatro grandes niveles Según Goleman (2007)

Escaso nivel de Inteligencia emocional. Cuando uno tiene poca inteligencia emocional lo que significa que uno debe desarrollar ejercicios y juegos. Las que se aprende y uno no se sienta mal pero sí considera aprender sobre esta gran estrategia, le permita conocer más a quienes le rodean.

Moderado nivel de Inteligencia emocional. Cuando uno sabe que puede controlar sus emociones. Sin embargo, uno puede mejorar, lo que el conocimiento de otros nos hace más ricos y nos fortalece. Además, las personas valorarán el esfuerzo, por tanto uno no debe rendirse.

Elevado nivel de inteligencia emocional. Cuando la persona sabe reconocer cuando otras personas están tristes. Puede leer el lenguaje corporal, así como actuar frente a cada situación. Recordando que es en una herramienta muy valorable. Ello enseña a otros sobre la inteligencia emocional y nunca deja de sentir empatía por su entorno.

Nivel Superdotado de inteligencia emocional. Cuando uno alcanza el top de la inteligencia emocional. Tal vez seas una persona con una gran comprensión de lo que ocurre en tu entorno. Ello te conduce a ser muy sensible en ciertas ocasiones, pero tú sabes cómo responder ante esto. Es considerado como una perla y el entorno, valora lo que tu empatía y enseña con el ejemplo aquello que ya eres naturalmente. 
Por tanto, la inteligencia emocional se relaciona con varias facetas del éxito personal y social como son el bienestar psicológico, el rendimiento académico entre otras, dichas emociones y su regulación se logran aprender desde la infancia y que éstas estan influenciadas por el contexto más próximo. El presente estudio tuvo por objetivo determinar la relación entre los estilos parentales e Inteligencia emocional de los padres y la IE de sus hijos/as en edades tempranas.

Muchos son los estudios que se han analizados sobre los efectos del estilo de crianza en el desarrollo emocional y el comportamiento de los hijos, éstos estilos parentales predicen sobre la competencia socioemocional, y éstas particularmente al estilo parental paterno la que se relaciona con las funciones psicosociales del hijo y con los sentimientos de soledad y depresión (McDowell, Parker y Wang, 2003). Los estilos parentales autoritarios se han asociado a desordenes obsesivo-compulsivos (Timpano, et al., 2010). Muchas veces se ha tratado de asociar éste estilo autoritativo - democrático, a una menor depresión en adolescentes (Liem, Cavell y Lustig, 2010). En el estudio de hechos emocionals con adolescentes disruptivos muestran que el estilo autoritario es el menos efectivo para mantener a estos chicos alejados de problemas (Pezzella, 2010). Así como el estilo maternal permisivo se ha asociado al comportamiento antisocial de forma directa e indirecta, por su efecto en el desarrollo de la empatía de los hijos (Schaffer, Clark y Jeglic, 2009). El estilo parental cálido se ha asociado fuertemente con la inteligencia emocional de los adolescentes y en el caso de los chicos, el estilo parental paterno predice la autoestima de los hijos (Zukauskiené, Malinauskiené y Erentaité, 2011).
La inteligencia emocional ha logrado relacionarse con varias facetas del éxito personal y social como el bienestar psicológico, así como el rendimiento académico, cuyo objetivo estudió la relación entre los estilos parentales e Inteligencia emocional de los padres, participado un total de 83 niños escolarizados donde 52 padres y 62 madres. Los resultados se relacionan con la Inteligencia emocional de los padres, con mayor inteligencia emocional tienden a mostrar un estilo democrático (Ramírez y Sainz, 2015).

Según La Organización Mundial de la Salud (2017), el CI es un indicador que agrupa varias "fotos fijas" sobre las habilidades cognitivas específicas son tomadas en un momento dado, es decir bajo unas determinadas condiciones, sobre determinados paisajes, lo que significa que el Coeficiente Intelectual, es un indicador muy útil cuando deriva de una prueba psicométrica, pero no es todo lo que necesitamos saber de la inteligencia, ni mucho menos todo lo que precisamos conocer de una persona. Se considera clave relativizar su valor y no considerarlo algo absoluto, predeterminado e inamovible del que se deriva que alguien "es" o "no es".

En el estudio sobre la Percepción y valoración de las emociones, de un total de 83 niños escolarizados en $2^{\circ}$ ciclo de educación infantil; 52 padres y 62 madres. Se han utilizado como medida de la Inteligencia Emocional del Cuestionario de BarOn y Parker (2002) dichos observadores, midieron las habilidades interpersonales, intrapersonales, y el manejo del estrés, adaptabilidad y estado de ánimo; (b) Prueba de Percepción y Valoración de las emociones (PERCERVAL, Mestre, et al., 2011), los que encontraron una percepción positiva. 
Los seres humanos con inteligencia moderada, logran constituir una franja de población relativamente amplia, lo que muchas veces se ven obligada a afrontar diversos obstáculos, incrementando el riesgo de dificultades a nivel educativo, social y en términos de salud mental (Gottfredson, 2005).

La inteligencia emocional, como concepto y como práctica, no parece cumplir las exigencias de cientificidad y precisión ya que, por un lado, se sitúan en el positivismo y por otro en el innatismo, ello las hace contradictorias y ambivalentes. Por tanto, con frecuencia el emocionalismo tiene que ver con la emoción como elemento de valoración del estado actual de una persona o de motivación para una acción critica en su mundo interior y exterior, dicha formación propone que los conceptos que aplica dependen por completo de la personalidad y el interés de los que la aplican (Manrique, 2015).

Respecto de la relación entre la Inteligencia emocional y la Satisfacción laboral concluye que existe una correlación positiva entre estas variables (Wen et al, 2019), las que señalan de quienes tienen control emocional, es que tienen un buen manejo de la inteligencia emocional, por tanto, la satisfacción laboral se ha relacionado más con el desempeño. (Bustamante et al 2020).

En base a lo citado, se puede decir que la inteligencia interpersonal, es la capacidad de entender a los demás e interactuar eficazmente con ellos. "Incluye la sensibilidad a expresiones faciales, la voz, los gestos y posturas y la habilidad para responder" (Gardner, 2000). Por otra parte, la inteligencia intrapersonal, de acuerdo a Campbell et al., (2000) "comprende nuestros pensamientos y sentimientos. En la medida en que podamos concientizarlos, más sólida será la relación entre nuestro mundo interior y el mundo exterior de la experiencia" (p. 3). Así también, Goleman (2007) manifiesta que "el desarrollo emocional intrapersonal, indica la manera de cómo la persona se maneja y se controla a sí mismo, de acuerdo con las herramientas adquiridas de su entorno, expresando sus sentimientos adecuadamente y con efectividad". (p. 106).

\section{MÉTODO}

La metodología que se aplicó fue de tipo descriptivo transversal con diseño no experimental, la población estuvo conformada por un total de 100 niños y niñas de las instituciones del nivel inicial de la zona rural aimara del ámbito de la UGEL Puno. La información se obtuvo con la aplicación de un cuestionario propuesto y diseñado por el test de Mesquite (Goleman, 2013), fundamentada, en conocer el nivel de inteligencia emocional, aquella que nace en la interacción con los padres de familia.

En esta metodología, las investigadoras se encuentran inmerso en el campo de estudio y de especialidad, con la finalidad de ver el nivel de Inteligencia emocional, la que se estructuró mediante el cuestionario sujeta a los ítems de medición de los niveles de inteligencia emocional, considerando 8 ítems, cada una con 4 alternativas y para la interpretación considerada de la siguiente manera:

Valoración de los resultados:

0 a 6 Puntos - Escasa inteligencia emocional 7 a 15 puntos - Inteligencia emocional moderada 15 a 25 puntos - Inteligencia emocional elevada 26 a 36 puntos - Inteligencia emocional superdotada 
Tabla 1. Test de mesquite.

\begin{tabular}{cccccccc}
\hline Ítem $\mathbf{1}$ & Ítem $\mathbf{2}$ & Ítem $\mathbf{3}$ & Ítem $\mathbf{4}$ & Ítem $\mathbf{5}$ & Ítem $\mathbf{6}$ & Ítem $\mathbf{7}$ & Ítem $\mathbf{8}$ \\
\hline 1.3 & 1.0 & 1.0 & 1.4 & 1.0 & 1.0 & 1.2 & 1.0 \\
2.0 & 2.1 & 2.2 & 2.0 & 2.4 & 2.2 & 2.0 & 2.2 \\
3.4 & 3.3 & 3.4 & 3.1 & 3.2 & 3.4 & 3.4 & 3.4 \\
4.0 & 4.4 & 4.0 & 4.2 & 4.0 & & & 4.1 \\
\hline
\end{tabular}

\section{RESULTADOS Y DISCUSIÓN}

Durante la pandemia, las familias vienen atravesando difíciles momentos por la pandemia del COVID 19, y Puno en Perú no está exenta a este problema familiar y se conoce que es muy importante conocer el nivel de Inteligencia emocional de los niños y niñas ya que ello estabiliza el accionar emotivo en las diferentes acciones, lo que puede ocasionar la aparición de variabilidad de la conducta social, crecen las agresiones verbales y físicas, la desadaptación social, problemas de aprendizaje, desadaptación y deserción escolar. En ese sentido, es necesario conocer el nivel de inteligencia emocional en niños y niñas del II Ciclo de Instituciones educativas del ámbito rural de la UGEL Puno, con ello se contribuirá a una relación estable y armoniosa entre padres e hijos, estudiantes y maestros y la buena adaptación social al contexto, el estudio coadyuva a un aprendizaje exitoso en el ambiente escolar que aún requieren fortalecer de manera adecuada el control emocional.

De acuerdo a los resultados obtenidos a través del test de Mesquite, adecuado a un cuestionario de google form, con el apoyo de los padres y madres de familia, el cual constó de 8 ítems de acuerdo al test, se consideró una de ellas para ver el sexo a la cual el niño pertenece, considerando la puntuación respectiva de cada ítem entre $(0,1,2$ y 4$)$; cuyos niveles considerados en el test, son: Escaso nivel de inteligencia emocional, moderado nivel de inteligencia emocional, elevada nivel de inteligencia emocional y superdotado nivel de inteligencia emocional, de un total de 100 padres de familia quienes intervinieron en este estudio.

Los resultados que se evidencian que en la Figura 1, señalan que de 100 niños y niñas del II Ciclo de Instituciones Educativas Rurales del ámbito de la UGEL Puno, 42 niños, que representa al 42\%, Espera que alguien le pregunte en privado del porqué de su enojo, seguido por 36 niños que representa al 36\% Intenta cambiar de actitud, en tanto 14 niños que representa el 14\% Continúa sin darle importancia a su enojo y seguido por 8 niños y niñas que representa al 8\%. Según el test de Mesquite para medir el nivel de Inteligencia Emocional predomina el nivel superdotado, aquel que representa la alternativa 3 , con un total de 42 niños que representa un $42 \%$, seguido por 36 niños que representa el 36\% (Nivel moderado), continuado por 14 niños que representa el 14\% (escasa inteligencia emocional) y 8 niños que representa un $8 \%$ No hace nada y de acuerdo a la valoración también se encuentran con escasa inteligencia emocional. 


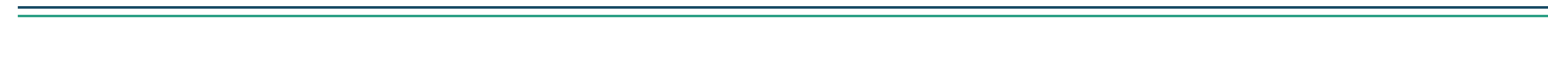

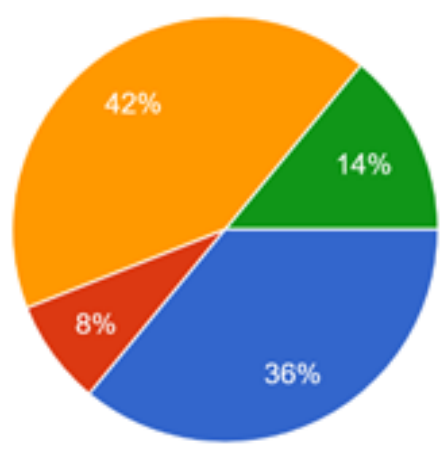

Intenta cambiar de actitud.

No hace nada.

Espera que alguien le pregunte en privado del porqué es su enojo.

Continúa sin darle importancia a su enojo.

Figura 1. Si te encuentras en una reunión familiar y de pronto tu niño o niña comienza a enojarse por alguna situación. ¿Qué hace tu niño o niña?

Los resultados evidencian en la Figura 2 que de 100 niños y niñas del II Ciclo de Instituciones Educativas Rurales del ámbito de la UGEL Puno, 36 niños y niñas que representa al $36 \%$, casi nunca le cuesta expresar lo que le pasa de acuerdo a la valoración del test indican que los niños y niñas se encuentran con inteligencia elevada, seguido por otros 36 niños que representa al 36\% casi siempre le cuesta expresar lo que le pasa ello indica que dichos niños y niñas tienen escasa inteligencia emocional, seguido por 16 niños y niñas que representa el $16 \%$ nunca les cuesta expresar lo que les pasa, lo que significa que tienen inteligencia emocional superdotada y sólo 12 niños y niñas que representa el $12 \%$ tienen escasa inteligencia emocional.

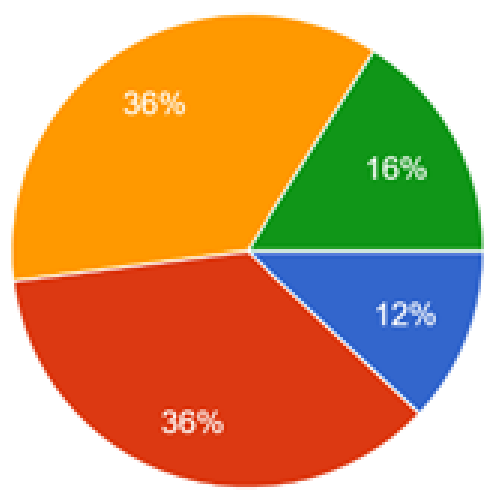

Siempre.

Casi siempre.

Casi nunca.

Nunca.

Figura 2. ¿Crees que tu niña o niño es una persona a la que le cuesta expresar lo que le pasa?

Los resultados que se evidencian en la Figura 3 destacan de 100 niños y niñas del II Ciclo de Instituciones Educativas Rurales del ámbito de la UGEL Puno, que 35 niños y niñas que representa al $35 \%$, dice lo que siente cuando se sienten angustiados o tristes y de acuerdo a la valoración del test indican que los niños y niñas se encuentran con inteligencia emocional moderada, seguido por otros 33 niños que representa al 33\% dice lo que siente o intenta decir, ello indica que dichos niños y niñas tienen una inteligencia emocional elevada, seguido por 24 niños y niñas que representa el $24 \%$ oculta lo que siente lo que significa que tienen una escasa inteligencia emocional y 8 niños y niñas que representa el $8 \%$ tienen no dice nada a nadie para que no se frían de él o de ella, lo que significa que también tienen una escasa inteligencia emocional. 


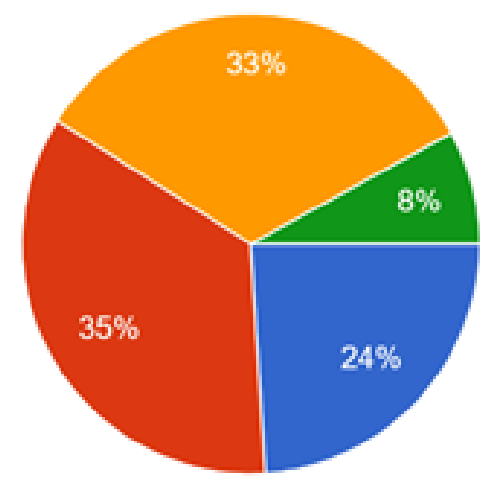

Oculta lo que siente.

Dice lo que siente.

Dice lo que siente e intenta resolver.

No dice nada a nadie para que no se rian de él/ella.

Figura 3. Cuando vez a tu niña o niño generalmente asustado o triste ¿Qué hace?

Los resultados que se evidencian en la Figura 4 mencionan que de 100 niños y niñas del II Ciclo de Instituciones Educativas Rurales del ámbito de la UGEL Puno, que 61 niños y niñas que representa al $61 \%$, dialoga con papá o mamá y comenta lo sucedido cuando se enoja de algo y de acuerdo a la valoración del test indican que dichos niños y niñas se encuentran con inteligencia emocional superdotada, seguido por otros 22 niños que representa al $22 \%$ se oculta de los demás para no hablar y prefiere que no lo molesten ello indica que dichos niños y niñas tienen una escasa inteligencia emocional, seguido por 9 niños y niñas que representa el $9 \%$ espera que algún familiar le pregunte de las situaciones de enojo lo que significa que tienen moderada inteligencia emocional y 8 niños y niñas que representa el $8 \%$ durante la noche recuerda lo ocurrido y sufre, lo que significa que también tienen una escasa inteligencia emocional.

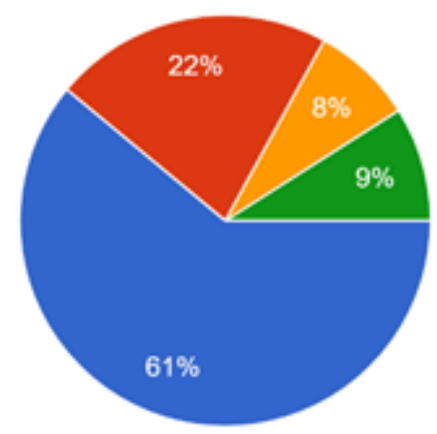

Habla contigo en privado y comenta lo sucedido.

Se oculta de los demás para no hablar y prefiere que no lo molesten.

Durante la noche recuerda lo ocurrido y sufre.

Le pregunta a algủn familiar qué hacer en situaciones de enojo.

Figura 4. Cuando tu niño o niña se siente enojado injustamente contigo. Generalmente ¿Qué hace?

Los resultados evidenciaron en la Figura 5 que de 100 niños y niñas del II Ciclo de Instituciones Educativas Rurales del ámbito de la UGEL Puno, que 52 niños y niñas que representa al 52\%, dialoga con papá o mamá frente a su enojo y de acuerdo a la valoración del test indican que dichos niños y niñas se encuentran con una moderada inteligencia emocional, seguido por otros 28 niños que representa al $28 \%$ Habla lo que cree necesario y sin límites ello indica que dichos niños y niñas tienen una inteligencia emocional superdotada, seguido por 12 niños y niñas que representa el $12 \%$ esconden sus sentimientos o pensamientos frente a su enojo lo que significa que tienen escasa inteligencia emocional y 8 niños y niñas que representa el $8 \%$ nunca te dice ni te pregunta nada, lo que significa que también tienen una escasa inteligencia emocional. 


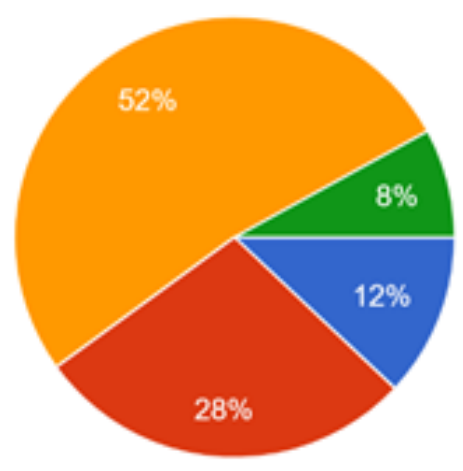

Esconde sus sentimientos y/o pensamientos.

Habla lo que cree necesario y sin limites.

Habla contigo sobre sus angustias o problemas.

Nunca te dice ni te pregunta nada.

Figura 5. Tú como papá o mamá, frente a una situación de enojo, generalmente tu niño o niña.

En la Figura 6 se indica que de 100 niños y niñas del II Ciclo de Instituciones Educativas Rurales del ámbito de la UGEL Puno, que 40 niños y niñas que representa al $40 \%$, espera que terminen los demás y recién participa en aquello que tenga que decir y de acuerdo a la valoración del test indican que dichos niños y se encuentran con una inteligencia emocional superdotada, seguido por otros 30 niños que representa al $30 \%$ comienza a caminar ansiosamente ello indica que dichos niños y niñas tienen una inteligencia emocional moderada, seguido por otros 30 niños y niñas que representa el 30\% presenta impaciencia lo que significa que tienen una escasa inteligencia emocional.

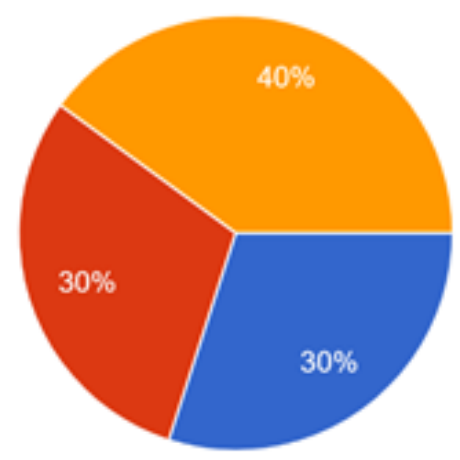

Que presenta impaciencia.

Comienza a caminar ansiosamente.

Espera que terminen los demás y recién participa en aquello que tenga que decir.

Figura 6. Cuando tu niño o niña quiere participar en alguna actividad.

Los resultados mostraron que de 100 niños y niñas del II Ciclo de Instituciones Educativas Rurales del ámbito de la UGEL Puno, que 53 niños y niñas que representa al 53\%, pide disculpas frente a un error cometido y de acuerdo a la valoración del test indican que dichos niños y niñas tienen una inteligencia emocional moderada, seguido por otros 35 niños y niñas que representa el 35\% se arrepiente y pide perdón a quién corresponda lo que significa que dicho grupo tienen una inteligencia emocional superdotada, seguido por 12 niños y niñas que representa el $12 \%$ no siente nada de malo frente a un error cometido, y de acuerdo a la valoración dichos niños no logran manejar su inteligencia emocional. (Figura 7). 


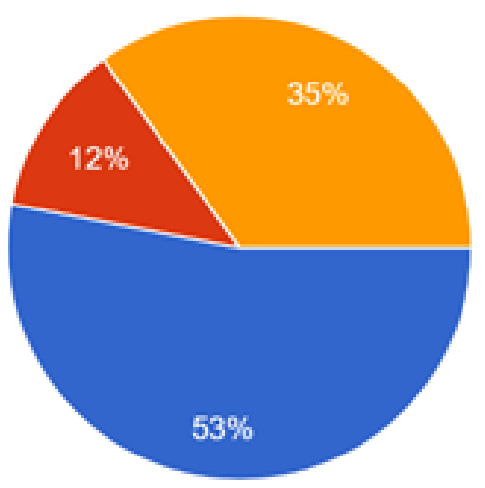

Pide disculpas y reconoce su error.

No siente nada de malo.

Se arrepiente y pide perdón a quien corresponda.

Figura 7. Cuando tu niño o niña ha cometido un error.

Los resultados evidenciados en la Figura 8, denotan que el 100 niños y niñas del II Ciclo de Instituciones Educativas Rurales del ámbito de la UGEL Puno, que 68 niños y niñas que representa el 68\%, pregunta qué le ha pasado cuando ve a un familiar desconsolado o llorando y de acuerdo a la valoración del test indican que dichos niños y niñas se encuentran con una inteligencia emocional superdotada, seguido por otros 17 niños que representa al $17 \%$ se sienta a su lado y simplemente espera que su familiar se calme cuando está desconsolado y llorando, ello indica que dichos niños y niñas tienen una moderada inteligencia emocional, seguido por 11 niños y niñas que representa el $11 \%$ solamente le mira a su familiar que está desconsolado y llorando lo que significa que tienen escasa inteligencia emocional y 4 niños y niñas que representa el $4 \%$ le pregunta insistentemente cada 5 minutos qué es lo que le ocurre lo que significa que también tienen una escasa inteligencia emocional.

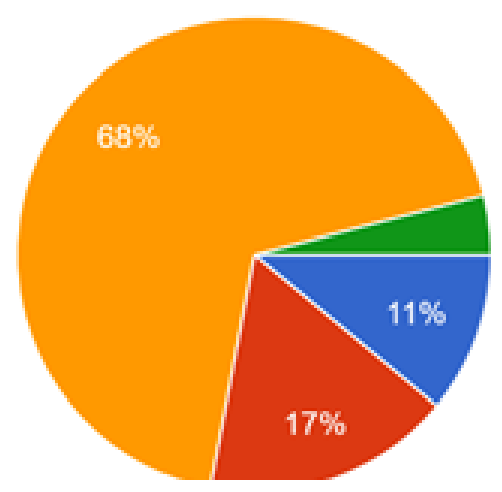

Solamente le mira.

Se sientas a su lado y simplemente espera que se calme.

Le pregunta que le ha pasado y trata de consolar.

Le pregunta insistentemente cada 5 minuto que es lo que le ocurre.

Figura 8. Cuando tu niño o niña, ve a otro familiar completamente desconsolado y llorando. ¿Qué hace? 


\section{Discusión}

En primer lugar hacer referencia al constructo de la inteligencia emocional y su importancia en el manejo y control adecuado de aquellas destrezas que permiten realizar tareas de manera eficaz, como la de percibir adecuadamente los estados emocionales asumiéndolos y expresando en un determinado contexto o situación; comprender la naturaleza de los estados emocionales y regular dichos estados impidiendo sus efectos negativos, dando mayor importancia a los aspectos positivos y ser capaces de hacer lo mismo con los estados emocionales de las personas que nos rodean (Ugarriza 2001). El nivel de inteligencia emocional, implica asumir un reto en éstos tiempos de pandemia, de acuerdo a los resultados obtenidos en el primer ítem predominó el nivel de inteligencia emocional superdotado con un $42 \%$ es así que de acuerdo a Goleman (2007) con su test Mesquite, dichos niños han logrado alcanzar el top de la inteligencia emocional, donde el niño comprende de lo que ocurre en su entorno. Esto le puede llevar a ser muy sensible en ciertas ocasiones, pero él sabe cómo responder frente a una situación de enojo de un familiar. En lo referente al ítem 2; ¿Crees que tu niña o niño es una persona a la que le cuesta expresar lo que le pasa?, predominó el de inteligencia emocional moderado, lo que significa que dicha inteligencia emocional la lleva internamente, sin embargo, puede mejorar, ya que ello fortalecerá el manejo emocional de sí mismo

En torno al ítem 3, Cuando vez a tu niña o niño, generalmente angustiado o triste ¿Qué hace?, en las respuestas predomina el nivel de inteligencia moderado, según Gottfredson (2005), constituyen una franja de población relativamente amplia, que se ve obligada a afrontar numerosos obstáculos a lo largo del ciclo de vida $\mathrm{y}$ esto se viene incrementando hacia el riesgo de dificultades a nivel educativo, social y en términos de salud mental. Además, de acuerdo al ítem 4, Cuando tu niño o niña se siente enojado injustamente contigo. Generalmente ¿Qué hace?, de acuerdo a los resultados obtenidos se observa que 61 niños que representa el $61 \%$ se encuentran en la inteligencia emocional superdotada, según la Organización Mundial de la Salud (2017), el CI es un indicador muy útil cuando deriva de una prueba psicométrica sólidamente construida, pero no es todo lo que requiere saber de la inteligencia, ni mucho menos todo lo que se precisa conocer de una persona. Es clave relativizar su valor y no considerarlo algo absoluto, predeterminado e inamovible del que se deriva que alguien "es" o "no es". Cabe señalar que de acuerdo al ítem 5 Tú como papá o mamá, frente a una situación de enojo, generalmente tu niño o niña, respondieron de 100 niños 52 de ellos que representa el $52 \%$ se encuentra en el nivel moderado, considerando que según Gottfredson (2005), constituye una población relativamente amplia, que se ve obligada a afrontar numerosos obstáculos a lo largo del ciclo de vida pues es necesario atender a este grupo de niños para mejorar y tener un buen control emocional. En el ítem 6 Cuando tu niño o niña quiere participar en alguna actividad, de los resultados obtenidos predomina que el $40 \%$ de niños y niñas se encuentran con una inteligencia superdotada, ya que los niños y niñas espera que terminen los demás y recién participan en aquello que tenga que decir, con ello según la OMS (2017), relativiza su valor y no lo considera como algo absoluto, ya que ello puede variar según las circunstancias. Y en el ítem 7 Cuando tu niño 
o niña ha cometido un error, en los resultados predomina la inteligencia emocional moderada, ya que dichos niños y niñas Se arrepienten y piden perdón o disculpa a quien corresponda por alguna acción cometida. Finalmente, en el ítem 8 Cuando tu niño o niña, ve a otro familiar completamente desconsolado y llorando. ¿Qué hace?, en dichos resultados predomina que, de 100 niños, 68 de ellos que representa el $68 \%$ se encuentran con inteligencia emocional superdotada, es decir manejan de manera adecuada su estado emocional.

Con dichos resultados de interpreta que el estilo parental predice la competencia socioemocional de los hijos, y particularmente el estilo parental paterno se relaciona con las funciones psicosociales del hijo y con los sentimientos de soledad y depresión (McDowell, Parker y Wang, 2003), es así que dichos niños del II Ciclo de las Instituciones Educativas de la zona rural del ámbito de la UGEL Puno, durante la pandemia se ubican en los niveles de Inteligencia emocional moderado a una inteligencia emocional superdotada, dicha formación que propone y los conceptos que aplica acaban por depender por completo de la personalidad y el interés de los que la aplican (Manrique, 2015).

La educación actual durante la pandemia es un camino para lograr una educación en valores, que provienen desde casa, direccionada a la igualdad de oportunidades. La pandemia del COVID-19 ha desnudado las acumuladas carencias y brechas sociales, pero a la vez, puso a prueba la capacidad de resiliencia, la renovación del sistema educativo peruano y por ende el desarrollo de la Inteligencia emocional. Es por ello, durante la pandemia los actores educativos han redoblado esfuerzos para garantizar la prestación del servicio educativo. En la actualidad se vive un escenario de aprendizajes individuales y colectivos, en el que combinar la salud, la vida y la educación pasa por actos de cooperación y de ayuda mutua. La educación prepara para la vida y también para lo inesperado, así los maestros desarrollan competencias y capacidades para actuar en cualquier contexto, compartiendo conocimientos, habilidades y actitudes.

Por otro lado, se ha tratado de asociar un estilo autoritario - democrático a una menor depresión en niños y niñas (Liem, Cavell y Lustig, 2010). Cabe señalar que disruptivos estilos muestran que es menor efectivo para mantener a estos chicos alejados de problemas del manejo de la inteligencia emocional, no se viene trabajando de manera adecuada, por el desconocimiento de estrategias en su manejo (Pezzella, 2010). El estilo maternal permisivo se ha asociado al comportamiento antisocial de forma directa e indirecta, por su efecto en el desarrollo de la empatía de los hijos (Schaffer, Clark y Jeglic, 2009). El estilo parental cálido se ha asociado fuertemente con la inteligencia emocional de los adolescentes y en el caso de los chicos, el estilo parental paterno predice la autoestima de los hijos, sin embargo, se deja de lado el manejo adecuado del control emocional de los niños y niñas, más aún durante la pandemia actual en la que venimos afrontando (Zukauskiené, Malinauskiené y Erentaité, 2011).

\section{CONCLUSIONES}

En el estudio se ha encontrado un nivel elevado en las puntuaciones emocionales, de la muestra de 100 niños, en términos generales significa que dichos niños y niñas saben reconocer sus emociones y actúan en base a cada situación, 
nunca dejan de sentir empatía por los demás. En los resultados obtenidos no se encontró diferencias estadísticas significativas a comparación con otros estudios. Es así que de acuerdo a los resultados se puede identificar que la Inteligencia Emocional de los niños y niñas son identificados de manera oportuna. Por tanto, la inteligencia emocional encontrado en los niños y niñas, permite identificar una mejor adaptación a los diversos ambientes escolares, familiares, sociales, entre otros. Es decir, es importante que los niños presenten una buena Inteligencia Emocional, ya que ello podrá evitar ciertas patologías en su vida futura, y que es vital que los padres presten la atención a este proceso a fin de ayudar a sus hijos y orientarlos de la mejor manera para autorregular sus emociones en este ámbito de sus vidas, como en todos los demás, tendrá un efecto positivo dentro de su desarrollo integral, por tanto éstos se conviertan en adultos felices, relajados, responsables, plenos, entre otras muchas cualidades. Finalmente queremos darte las gracias y esperamos que la información aquí proporcionada te sea de gran utilidad.

\section{REFERENCIAS}

Bustamante, M. A., Álvarez, A. J., Villalobos, M. E., y Lucero, M. I. (2020) Percepción de la calidad de vida laboral de los trabajadores de los centros de salud familiar de la zona central de Chile, http:// dx.doi.org/10.4067/S0718-07642020000300065, Información tecnológica, 31(3), 65-74

Campbell, L., Campbell, B., y Dickenson, D. (2000). Inteligencias Múltiples. Troquel

Gardner, H. (2000). Mentes Extraordinarias. Kaiross

Goleman, D. (2007). Inteligencia Emocional. Batann Books

Goleman, D., (2013), Focus, el motor interno de la excelencia. Nueva York, Estados Unidos: ediciones en español. Ediciones B, argentina S.A. 2014
Gottfredson, L.S. (2005). Implications of cognitive differences for schooling within diverse societies. In: C.L. Frisby \& C.R. Reynolds (eds.), Comprehensive handbook of multicultural school psychology. Hoboken, NJ: Wiley \& Sons https://webcache.googleusercontent. com/search?q=cache:Z2chEnhTCv8J:https:// p s i s e m a d rid.org/inteligencia limite $/+\& c d=7 \& h l=e s-419 \& c t=c l n k \& g l=$ pe .

Liem, J. H., Cavell, E. C. y Lustig, K. (2010). The influence of authoritative parenting during adolescence on depressive symptoms in young adulthood: Examining the mediating roles of self-development and peer support. The Journal of Genetic Psychology, 171, 73-92

Manrique Solana, R. (2015). La cuestión de la inteligencia emocional. Revista de la Asociación Española de Neuropsiquiatría, 35(128), 801814. https://dx.doi.org/10.4321/S021157352015000400008

McDowell, D., Parker, R. y Wang, S. (2003). Differences between mothers and fathers advicegiving style and content: Relations with social competence and psychological functioning in middle childhood. Merrill-Palmer Quarterly, 49 (1), 55-76

Mestre, J. M., Guil, R., Martínez-Cabañas, F., Larrán, C. y González, G. (2011). Validación de una prueba para evaluar la capacidad de percibir, expresar y valorar emociones en niños de la etapa infantil. REIFOP, 14(3)

Organización Mundial de la Salud. (30 de marzo de 2017), El coeficiente intelectual. Recuperado de: https://incansableaspersor.wordpress. com/2017/03/30/el-mito-de-la-oms-y-el-ci130-para-ser-superdotado/.

Pezzella, F. S. (2010). Authoritarian parenting: A race socialization protective factor that deters African American adolescents from delinquency and violence (Doctoral dissertation). Recuperado de la base de datos ProQuest Dissertations and Theses database. (UMI No. 3398173)

Ramírez-Lucas, A., Ferrando, M., \& Sainz, A. (2015) ¿Influyen los Estilos Parentales y la Inteligencia Emocional de los Padres en el Desarrollo 
Emocional de sus Hijos Escolarizados en $2^{\circ} \mathrm{Ciclo}$ de Educación Infantil? Acción Psicológica,12(1), 65-78. https://dx.doi.org/10.5944/ap.12.1.14314

Schaffer, M., Clark, S. y Jeglic, E. L. (2009). The role of empathy and parenting style in the development of antisocial behaviors. Crime \& Delinquency, 55, 586-599. doi: 10. 1177/0011128708321359

Timpano, K. R., Keough, M. E., Mahaffey, B., Schmidt, N. B. y Abramowitz, J. (2010). Parenting and obsessive compulsive symptoms: Implications of authoritarian parenting. Journal of Cognitive Psychotherapy: An International Quarterly, 24(3), 151-164. doi: 10. 1891/08898391.24.3.151
Wen, J., Huang, S. S., y Hou, P. (2019) Emotional intelligence, emotional labor, perceived organizational support, and job satisfaction: A moderated mediation model, https://doi. org/10.1016/j.ijhm.2019.01.009, International Journal of Hospitality Management, 81, 120-130

Zukauskiené, R., Malinauskiené, O. y Erentaité, R. (2011). Effects of parenting styles and emotional intelligence on self-efficacy and selfesteem in late adolescence: gender differences. Psichologija, Journal of Vilnus University 